\title{
MITOLOGÍA Y CIENCIA FICCIÓN: EL CASO DE MYTHS OF THE NEAR FUTURE, DE JAMES G. BALLARD
}

\author{
MYTHOLOGY AND SCIENCE FICTION: \\ THE CASE OF JAMES G. BALLARD'S MYTHS OF THE NEAR FUTURE
}

\author{
Juan VARO ZAFRA \\ Universidad de Granada \\ juanvaro@ugr.es
}

\begin{abstract}
Resumen: La relación entre mitología y ciencia ficción es paradójica: si, teóricamente, la ciencia ficción se presenta como opuesta del mito; en su producción narrativa recurre frecuentemente a personajes y esquemas míticos, materializando su dimensión prospectiva a través de la actualización evemerista o alegórica de mitos. Este trabajo revisa críticamente los presupuestos teóricos que escinden la literatura de ciencia ficción de los relatos míticos y la literatura fantástica. A continuación, analizaremos el modo en que James G. Ballard afronta esta cuestión en su narrativa breve, particularmente en Myths of the Near Future, que sobrepasa estas diferencias y plantea un nuevo marco teórico común entre literatura fantástica y mítica y la ciencia ficción.
\end{abstract}

Palabras clave: Ciencia ficción. James G. Ballard. Myths of the Near Future. Mito.

\begin{abstract}
The relationship between mythology and science fiction is paradoxical: if, theoretically, science fiction is presented as the opposite of myth; in its narrative production, science fiction frequently resorts to mythical characters and schemes, materializing their prospective dimension through the evemerist or allegorical updating of myths. This work critically reviews the theoretical assumptions that divide science fiction literature from mythical stories and fantasy fiction. Next, it analyzes the way in which James G. Ballard addresses this question in his short narrative, particularly in Myths of the Near Future, which goes beyond these differences and raises a new common theoretical framework between fantasy and mythical literature and science fiction
\end{abstract}

Keywords: Science Fiction. James G. Ballard. Myths of the Near Future. Myth.

\section{MITO Y CIENCIA FICCIÓN: APUNTES PARA UNA REFORMULACIÓN CONCEPTUAL}

Desde sus inicios, la ciencia ficción se esforzó en distanciarse del género fantástico, estableciendo un paradigma opuesto ya reconocible en la definición que Robert A. Heinlein enunció en 1959: 
Realistic speculation about possible future events, based solidly on adequate knowledge of the real world, past and present, and on a thorough understanding of the nature and significance of the scientific method.

To make this definition cover all science fiction (instead of "almost all") it is necessary only to strike out the word "future" (Heinlein, 1959: 22-23).

Exposición realista, cabe entender verosimilitud (Jameson, 2005: 86), orientación prospectiva y cientifismo son las coordenadas fundamentales de la ciencia ficción ${ }^{1}$.

Tal vez sea Darko Suvin el teórico que más taxativamente haya defendido la dimensión cognoscitiva de la ciencia ficción a través de dos conceptos determinantes: el estrangement y el novum: "SF is, then, a literary genre whose necessary and sufficient conditions are the presence and interaction of estrangement and cognition, and whose main formal device is an imaginative framework alternative to the author's empirical environment" (Suvin, 2016: 20). De este modo, el extrañamiento deslinda la ciencia ficción de la literatura realista, mientras que el elemento cognoscitivo la distingue del relato mítico, el cuento de hadas y la literatura fantástica ${ }^{2}$.

El novum debe ser científicamente verosímil: "A novum of cognitive innovation is a totalizing phenomenon or relationship deviating from the author's and implied reader's norm of reality [...] The novum is postulated on and validated by the post-Cartesian and post-Baconian scientific method" (Suvin, 2016: 80-81).

Suvin propone así una definición, todavía considerada referencial: “An SF narration is a fiction in which the SF element or aspect, the novum, is hegemonic, that is, so central and significant that it determines the whole narrative logic —or at least the overriding narrative logic - regardless of any impurities that might be present" (Suvin, 2016: 87). Aunque reconoce que algunos rasgos de la ciencia ficción son mitomórficos, Suvin rechaza cualquier ulterior analogía (Suvin, 2016: 39-40).

La concepción posmoderna de la ciencia ficción, a partir, especialmente, de los trabajos teóricos de Samuel Delany, ha subrayado la singularidad lingüística del género, hasta el punto de considerarlo paraliterario (Jameson, 2005: 91; Broderick, 1995: 28, 70-

\footnotetext{
${ }^{1}$ Hay otros intentos de describir el género. Marc Angenot lo consideró como una construcción semiótica a partir del extrañamiento. Este se produce en diversos niveles: 1. Signo/Referente/Paradigma: el discurso de la ciencia ficción es un discurso carente de referente, puramente conjetural. Es cierto que este rasgo puede darse también en la literatura realista, pero en la ciencia ficción, el objetivo estético consiste en crear un mundo remoto y, sin embargo, inteligible. El lector debe abandonar las reglas que rigen su mundo empírico, y asumir una inteligibilidad paradigmática engañosa que opera como un espejismo en la comprensión del texto, utópica en el sentido estricto del término. La ciencia ficción no se define por ninguna relación directa entre su mundo ficticio y el mundo empírico, sino por las relaciones inherentes al discurso ficticio entre sintagma y paradigma, a través de palabras ficticias que conforman una "exolingüística": lenguas extraterrestres o del futuro que suponen un paradigma ausente, un algo "no dicho", y de cuya existencia es persuadido el lector en la "suspensión de la incredulidad" de la lectura (Angenot: 1979). El problema de esta descripción es que es también válida para cualquier subgénero del fantástico. Sobre esta cuestión, como se apuntará más abajo, profundizará la teoría posmoderna del género. Los artículos de Science Fiction Studies en su edición digital no están paginados.

${ }^{2}$ Para un resumen de las críticas a Suvin, véase Broderick (1995: 31-34).
} 
74), incidiendo, por una parte, en los contenidos verbales del novum y el extrañamiento, esto es, la producción de significantes opacos que carecen de significados definidos y que generan paradigmas vacíos; $\mathrm{y}$, por otra, en la esencial subjuntividad del género, su carácter hipotético, al margen de la literatura mimética realista (Broderick, 1995: 67-69). Próxima a este concepto, Noemí Novell Monroy apunta al carácter conjetural como rasgo determinante

como un género que se pregunta what if, $\mathrm{y}$ tras ello construye sus mundos [...] La diferencia de la CF recae en su naturaleza conjetural y extrapolativa: su tipo de actividad mimética se podría llamar conjetural en tanto que es una copia, una imitación que parte de una realidad empírica [...] y la extrapola hacia el futuro o especula sobre ella en sus condiciones actuales (Novell Monroy, 2008: 143-144).

Para Fernando Ángel Moreno, la ciencia ficción y el género fantástico contemplan el campo referencial interno del texto como una transgresión de los principios de funcionamiento del campo referencial externo,

Pero mientras en el fantástico la transgresión problematiza la realidad [...] en la ciencia ficción la realidad no se muestra problemática [...] Lo que se problematiza es la lectura que hacemos de la realidad y todo lo que hemos construido a partir de dicha lectura errónea $[\ldots]$ lo que se plantea la ciencia ficción es romper apriorismos sociales, no físicos ni naturales, como hace el fantástico (Moreno, 2009: 77-78).

En nuestra opinión, la exigencia de cientificidad constituye no tanto un elemento estructural del género cuanto un elemento natural de la retórica de su discurso. El cientifismo es una estrategia persuasiva (acerca de la apariencia del carácter prospectivo del género), y un mecanismo para justificar verosímilmente episodios, que, de otro, modo, serían leídos como aventuras fantásticas. Lo que constituye el requisito esencial de la ciencia ficción es la problematización de la experiencia humana del progreso científico y técnico presente o especulativo.

Si la cientificidad debe ser reconsiderada como un rasgo de la retórica del género, también su dimensión prospectiva debe ser cuestionada. En sus comienzos, la orientación hacia el futuro se ubicaba en el contexto del descubrimiento ilustrado del progreso científico y social. El cuestionamiento de este progreso con la descomposición de las viejas utopías a tenor del colapso del siglo XX no dejaba de ser una inversión del paradigma que en nada afectaba al carácter prospectivo del género porque las distopías eran también utopías, si bien de signo contrario (Jameson, 2005: 216-221).

Ahora bien, lo que hace tambalear la prospectiva literaria de la ciencia ficción no es tanto el temor al futuro como su desvanecimiento, consecuencia de un progreso transformado en una continua auto-justificación del presente, mediante un futuro que el mismo se da (Blumenberg, 2008: 41; Berardi, 2014: 33-41, 114; Greenland, 2012: 9). Quizá como reacción a esta clausura del futuro, algunas obras recientes han desarrollado sus tramas en un futuro remotísimo, alterando la noción misma de lo prospectivo 
mediante una expansión del extrañamiento que conduce a la frontera de lo fantástico, en cuanto lo verosímil aparece como inconcebible. Tal es el caso de las novelas postsingularistas en las que un acontecimiento extraordinario rompe la continuidad del progreso tecnológico, bien frenándolo, bien apresurándolo, desarrollando unas tecnologías futuras improbables cuyo único límite es precisamente lo fantástico.

Para James G. Ballard, el concepto de prospectiva es consustancial al género, si bien de un modo particular: "For me, science fiction is above all a prospective form of narrative fiction; it is concerned with seeing the present in terms of the inmediate future rather than the past" (Brigg, 1985: 13). En vez de proyectar aspectos de la realidad presente o incluso pasada del mundo empírico al futuro ficcional, trata de explicar el presente en términos del futuro inmediato: un presente que absorbería el pasado y el futuro en un momento simultáneo caracterizado por el estancamiento y el narcisismo, una situación en la que el progreso científico se convierte en rutina (Sánchez Fernández, 2018: 92).

La relación entre ciencia ficción y los géneros de lo fantástico debe, por tanto, reformularse, no solo porque la ciencia ficción invade continuamente el espacio del weird, sino porque lo fantástico ha adoptado fórmulas que exceden lo sobrenatural y se dirigen hacia el descubrimiento de una realidad más compleja y profunda que hace suyos lo extraño y lo ilógico (Alazraki, 2001: 276-277). Esta reformulación de lo fantástico crea un limes habitable para ambos géneros, un espacio de intersección con la ciencia ficción en creciente expansión debido a la cada vez mayor confusión entre lo fantástico y lo científico en su modalidad especulativa ${ }^{3}$.

Desde estos postulados, consideramos la ciencia ficción como el género que explora y problematiza el nuevo mundo surgido de la crisis del humanismo, en la modalidad abierta por el desarrollo científico técnico, la posibilidad de una pluralidad de mundos, la apertura de un tiempo futuro no delimitado por la esperanza escatológica, y, al mismo tiempo, la conciencia de las limitaciones de estas perspectivas, la nostalgia de la verdad del mundo griego y del mundo cristiano medieval (verdad moral pero sobre todo verdad ontológica), y la angustia producida por la conciencia de acabamiento de lo humano y su sentido. En este sentido, y respecto a la relación con el fantástico, Fredric Jameson, afirma:

\footnotetext{
${ }^{3}$ David Roas ha explicado cómo afectan al fantástico los nuevos paradigmas de la realidad a partir de los avances de la física cuántica (Roas, 2011: 21-28). Por otra parte, como anota Campra, la clasificación de lo fantástico de Ana Barrenechea (1972) dejaba abierta la puerta a la confluencia con la ciencia ficción al distinguir dos subórdenes del fantástico según la semántica global del texto: el formado por los textos en los que la existencia de otros mundos no pone en duda la existencia del nuestro; y el formado por textos que postulan la realidad de lo que en primera instancia se formulaba como imaginario, y viceversa, de tal modo que se duda de la existencia del propio mundo. En esta propuesta no se distingue bien entre lo fantástico y algunos motivos de la ciencia ficción, como por ejemplo la existencia de mundos paralelos (Campra, 2008: 25-26). Así, respecto a obras como La invención de Morel de Adolfo Bioy Casares, Rosalba Campra observa: "La utilización de este tipo de motivos ha hecho sin duda menos discernible el límite entre los textos que consideramos fantásticos y los de ciencia ficción, pero manteniendo para los primeros, de todos modos, un aura inquietante e inexplicable a pesar de la referencia científica (o pseudocientífica)" (Campra, 2008: 56).
} 
Si la ciencia ficción constituye la exploración de todas las restricciones arrojadas por la propia historia - la red de contrafinalidades y antidialécticas producidas por la propia producción humana-, la fantasía es el otro lado de la moneda y una celebración de la capacidad y la libertad creativas de los humanos, que solo se vuelve idealista mediante la omisión de esas restricciones materiales e históricas (Jameson, 2005: 89).

Por estas razones, las vías abiertas por James G. Ballard, tanto en la exploración del Inner Space 4 como en la permuta de la pregunta clásica de la ciencia ficción What if? por la más urgente What is? suponen un giro copernicano, resultado de los cambios que respecto al género y su relación con la ciencia, lo fantástico y la prospectiva hemos examinado. Este What is? se concreta en la proposición de hipótesis sobre la vida presente en relatos ubicados en un futuro inmediato que sirven de ejemplos o ensayos de la misma (Ballard, 2014d: 74-75). A medida que este planteamiento va asentándose, las novelas de Ballard (más que los relatos) se van haciendo más ensayísticas, con extensos y abundantes excursos explicativos a cargo de personajes ambiguos, con frecuencia psiquiatras, como los doctores Nathan (The Atrocity...), Penrose (Super-Cannes) o Maxted (Kingdom Come).

Así, la ciencia ficción no solo gana un universo nuevo que debe ser cartografiado, el Inner Space, sino que desliza la retórica del discurso literario hacia las miradas subjetivas del autor y del lector. No es la ciencia sino el modo en que esta problematiza la vida cotidiana lo que ahora se convierte en el vórtice de fuerzas que animan el género, tal como expresa en "Fictions of Every Kind" [1971]:

The subjetc matter of science fiction is the subject matter of everyday life: the gleam on refrigerators cabinets, the contour of a wife's or husband's thighs passing the newsreel images on a colour TV set, the conjunction of musculature and chromium artefact within an automobile interior, the unique postures of passengers on an airport escalator (Ballard, 1997: 207).

Como corolario de todo lo expuesto, podemos establecer la siguiente distinción de los rasgos definitorios de la ciencia ficción: el elemento esencial consiste en la problematización de la experiencia humana del progreso técnico y científico; los elementos naturales son la especulación científica y la dimensión prospectiva. Lo prospectivo no es la proyección de la mirada del autor hacia el futuro sino la construcción de una mirada nueva, especulativa, sobre la realidad presente, con una retórica propia que la haga verosímil: el novum no radica en la presentación de nuevas realidades avaladas por el método científico, sino en la novedad de la mirada, producto de la relación compleja entre el individuo o la sociedad y el progreso científico, y en el discurso que la articula

\footnotetext{
${ }^{4}$ Ballard define el Inner Space como "an imaginary realm in which on the one hand the outer world of reality, and on the other the inner world of the mind meet and merge. Now, in the landscapes of the surrealist painters, for example, one sees the regions of Inner Space; and increasingly I believe that we will encounter in film and literature scenes which are neither solely realistic nor fantastic. In a sense, it will be a movement in the interzone between both spheres" (Ballard, 1968: 106). Véase también Wilson (2017: 5).
} 
en una nueva verosimilitud que ya no puede ser la del realismo narrativo, pero que pretende responder a un nuevo concepto de realidad.

En este contexto, la relación con lo fantástico se hace más compleja: lo fantástico y lo mítico dan cuerpo a realidades del espacio interior, en un imaginario expresado en formas oníricas o alucinatorias; espacios del deseo y la angustia que intuyen una experiencia simultánea del tiempo, nuevas formas de sexualidad o que son el resultante del colapso mental provocado por la intromisión de la tecnología y sus políticas.

De este modo, la incertidumbre del lector ante lo sobrenatural que, según Todorov, es el elemento vertebral del fantástico (Todorov, 2001a: 48, 59) ${ }^{5}$, o los vacíos causales en la trama (Campra, 2008: 130-133) o el conflicto entre lo real y lo imposible (Roas, 2011: 30-31) se reformulan en una clave delimitada por la situación comunicativa del relato, que impide así la confusión total de ambos géneros ${ }^{6}$. Esta reformulación se produce en los términos siguientes:

En primer lugar, la incertidumbre del lector no se resuelve, lo que cortocircuita el descenso a lo maravilloso tal como había considerado Todorov. El lector se enfrenta a una versión radical del extrañamiento o de la carencia de referentes propios apuntados por Darko Suvin y Marc Angenot. Porque en los experimentos más osados de William Burroughs, Philip K. Dick, Stanislav Lem, James G. Ballard o Greg Egan el lector carece incluso de un paradigma engañoso que le permita colmar el vacío referencial del relato ${ }^{7}$. Técnicas de vanguardia como el cut-up, el collage, la enumeración caótica al filo de la escritura automática, el contrapunto, el monólogo interior, las lenguas inventadas o puramente personales, las alteraciones cronológicas y causales y el perspectivismo invaden la ciencia ficción con el propósito de dar noticia fidedigna de la nueva realidad resultante de la experiencia del progreso científico-técnico.

En segundo lugar, estos autores emplean frecuentemente, como estrategia discursiva, la paradoja epidémica, es decir, la parodia o el pastiche de discursos procedentes de otros ámbitos, en estos casos científicos o técnicos (Broderick, 1995: 116), para sumergirse en un caos explicativo que, lejos de cumplir sus fines cognoscitivos, como requería Suvin,

\footnotetext{
5 Para una valoración crítica del concepto y clasificación de lo fantástico de Todorov, pueden verse Barrenechea (1972) y Campra (2008: 22-25 y 189-190).

${ }^{6}$ Todorov consideraba la ciencia ficción como un subgénero del fantástico maravilloso: lo maravilloso científico: "Aquí lo sobrenatural es explicado de una manera racional, pero a partir de leyes que la ciencia contemporánea no conoce [...] Se trata de relatos en los que, a partir de premisas irracionales, los hechos se encadenan de una forma perfectamente lógica" (Todorov, 2001b: 80); lo que generó una respuesta severa de Stanislaw Lem (Lem, 1974).

${ }^{7}$ Como apunta Rosalba Campra, tanto la ciencia ficción clásica como el fantástico tradicional generan paradigmas ex novo que explican, en mayor o menor medida, los acontecimientos de la trama. Sin embargo, hay casos en los que la ausencia de paradigma resulta irreductible. Campra denomina este supuesto como modulación sintáctica de lo fantástico: la carencia de motivación crea una fluctuación que afecta a la coherencia del mundo, aunque no sea posible identificar en sus contenidos algo más allá del orden natural (Cambra, 2008: 129-130). Algo similar sucede en los casos de ciencia ficción que aquí señalamos: no solo el extrañamiento se vuelve impenetrable, sino que el propio discurso se desconcierta, abundando en la confusión y quiebra del paradigma ex novo, más intuido que cierto.
} 
aumentan el vacío lógico y la desorientación interpretativa del lector, reduciendo toda la argumentación al absurdo.

En tercer lugar, los elementos sobrenaturales quedan subsumidos en la más amplia situación comunicativa de la narración: se presentan en forma de alucinación o sueño. La narración en primera persona es lo que da significado al mundo narrado; un relato dañado por las drogas y la locura, el trauma o la obsesión. En otras ocasiones, la narración en tercera persona a cargo de un narrador externo está sostenida por una voz poco fiable, bien porque deja intuir que estamos ante una falsificación de la realidad, bien porque el narrador no conoce nada del asunto, o traslada la visión de un focalizador personaje enajenado, o porque se encuentra él mismo perturbado, o porque la narración descansa sobre un documento o conjunto de documentos absurdos y paródicos.

Finalmente, nos encontramos ante relatos de ciencia ficción que enmarcan otros de carácter fantástico. En esta retórica del desconcierto, corresponde al lector conjeturar, a través de indicios (contradicciones, repeticiones, variantes, humor absurdo o violento, imágenes en apariencia arbitrarias, un tono frío, impasible y distanciado, etc.), el paradigma aludido que, frente a lo que exponía Angenoc, resulta no engañoso, sino verdadero, por cuanto no responde al What if? de la ciencia ficción clásica, sino al What is? que marca la renovación del género.

La incorporación de parámetros estilísticos como la ausencia de profundidad mimética o ilusoria, la pérdida o atenuación de la subjetividad, la sustitución del tiempo por el espacio como dimensión hegemónica, y el ya anunciado abandono de un único estilo en favor del pastiche o la escritura esquizofrénica ${ }^{8}$, y el collage conforman los rasgos más representativos de la ciencia ficción posmoderna (Jameson, 2005; Broderick, 1995).

El mito, como expresión de las fuerzas de la imaginación o como forma simbólica de conocimiento, adquiere un valor importante en la construcción de estos relatos, por su valor cognoscitivo, en la exploración del Inner Space: a la angustia o el trauma generados por la vis expansiva y totalizante de una realidad áspera, problematizada por la invasión agresiva e incomprensible de la ciencia y la técnica, el mito opone el proteico universo de las imágenes y el deseo; metáforas que ocupan el vacío de la angustia, como respuesta al horror vacui que esta produce (Blumenberg, 2003: 12-16, 47). Pero, al interiorizarse, el mito se hace trágico y lírico; de este modo, la épica de la ciencia ficción clásica, del outer space, deja lugar a una ciencia ficción crepuscular en la que el conflicto es decisivamente mental.

El mito ofrece al escritor de ciencia ficción un modelo de temporalidad que, en cierto modo, refleja mejor la experiencia no cronológica del tiempo (Blumenberg, 2003: 142); y también una jerarquía temporal que engendra tiempos de calidad, "aquellos momentos particularmente sagrados, de paradigmas que determinan las normas morales y las formas cultuales, lo que vale tanto como decir las estructuras fundamentales del mito"

\footnotetext{
${ }^{8}$ Para la escritura esquizofrénica en Philip K. Dick, véase Capanna (2006: 83-84).
} 
(Meletinski: 2001, 213). En consecuencia, el tiempo que engendra el mito no es esencialmente un pasado remoto, sino un tiempo pregnante que genera una durabilidad esencial, esto es, un futuro a partir de sí mismo. En este sentido dice Blumenberg que la originalidad del mito, más que con el pasado, tiene que ver con "la conservación de los contenidos desde la antigüedad remota hasta el presente en el que el mito se actualiza. Es la continuidad a través del tiempo lo que confiere a un contenido aquella cualidad que se atribuye a los orígenes, a la relación inmediata del tiempo original con todo lo experimentable" (Blumenberg, 2003: 177). Un mito del futuro es el tiempo original del futuro. Este es el sentido del título de Myths of the Near Future.

\section{LOS MITOS POR VENIR DE JAMES G. BALLARD}

La New Wave de la ciencia ficción británica se conformó en torno a la revista $\mathrm{New}$ Worlds, a partir de 1964, cuando Michael Moorcock se convirtió en su director. En sus editoriales, Moorcock reivindicaba una ciencia ficción innovadora en lo formal y agresiva en sus contenidos, alejándose de los temas y estilo de la ciencia ficción convencional. Junto a Moorcock, James G. Ballard, Brian Aldiss y John Brunner, entre otros, plantearon una ciencia ficción basada, a menudo, en los avances de la antropología, la psicología o la sociología más que en el avance científico técnico, generando inquietantes alegorías políticas y sociales, en el denso contexto histórico de la guerra de Vietnam, la explosión de las drogas alucinógenas, la Guerra Fría y la revolución sexual (Moreno, 2010: 374376); una ciencia ficción que pretendía ser la nueva literatura de la era espacial (Greenland, 2012: 32).

Los autores de New Worlds, se incorporaban así a la operación de renovación del género que Fredric Jameson ha denominado "etapa estética", en la que se incluirían también escritores norteamericanos como Philip K. Dick, Ursula Le Guin y Samuel Delany; "estética", en cuanto se interesaba fundamentalmente por los dilemas de la percepción y la representación, la condición del lenguaje y la problematización de lo real (Jameson, 2005: 122; Greenland, 2012: 166-168). La entropía, entendida no solo como degeneración de la energía, sino también como desintegración de la sociedad y de la conciencia individual se convierte en el tema dominante, muchas veces comprendido por los autores de New Worlds de forma gozosa o extática, como una suerte de posibilidad extrema de un nuevo comienzo (Greenland, 2012: 10, 192-193).

En esta profunda renovación del género, la obra de James G. Ballard cobra una significación especial. La decisiva, aunque no exclusiva, presencia del surrealismo ${ }^{9}$ en su obra motivó que Ballard se ubicara en la periferia de la ciencia ficción, al menos en lo relativo al papel jugado por la imaginación y su poder transformador subversivo individual y social (Baxter, 2008: 6-7). El surrealismo, junto al situacionismo, aportó a Ballard una epistemología, una ética, $\mathrm{y}$, sobre todo, una retórica de los lugares de raíz

\footnotetext{
${ }^{9}$ Para la presencia del surrealismo en la ciencia ficción, puede verse Parkinson (2015).
} 
psico-geográfica, inspirada en los paisajes de Ernst, Chirico, Delvaux y Dalí; junto a una retórica del tiempo cuyos instrumentos más reconocibles eran el fragmentarismo, el collage, la simultaneidad, la elipsis, las repeticiones y los catálogos. Denomino catálogo a uno de los hallazgos más genuinos de Ballard a partir de The Atrocity Exhibition: se trata de una variante de la enumeración caótica y del documental surrealista que consiste en la enumeración de una serie de objetos en un lugar determinado. A diferencia de la enumeración caótica, se trata de un corpus cerrado y adscrito a un lugar o a una persona, con frecuente intención irónica, tanto por lo inesperado del "objeto encontrado" respecto a la persona a la que se le atribuye, como por la relación desautomatizada que se genera entre los distintos componentes del catálogo. La relación dialéctica entre el catálogo y la persona o lugar al que corresponde lo distingue del "documental" surrealista.

Pero la aproximación al surrealismo no se limita a los paisajes pictóricos, al Inner Space o a los mencionados recursos formales, sino a la contemplación irónica, experimental, curiosa, de la subversión a través de la locura y la regresión antropológica. Los futuros ballardianos están tanto a cinco minutos del presente (Sellar, 2007) como del pasado más remoto.

El mito aparece en la obra de Ballard desde el comienzo. En un temprano artículo sobre William Burroughs, "Myth Maker of the Twentieth Century" publicado en New Worlds en 1964, Ballard proclama, contra el parecer general de autores, estudiosos y lectores del género: "Once it gets 'off the ground' into space, all science fiction is fantasy, and the more serious it tries to be, the more naturalistic, the greater its failure, as it completely lacks the moral authority and conviction of a literature won from experience" (Ballard, 1997: 129).

Sin embargo, en una entrevista de 1982, Ballard distingue entre surrealismo y fantasía y sugiere un límite a la relación entre lo fantástico y la ciencia ficción:

Eso es lo que siempre me atrajo de los surrealistas, que tenían el ojo interior. El ojo interior continúa siendo crítico; no se ha limitado a responder a la imaginación. Los surrealistas dirigen este ojo crítico también hacia sus propias fantasías; uno siente que todos esos pintores están despiertos, que todos esos sueños fueron soñados por durmientes que estaban despiertos. Esto es lo importante (Ballard, 2015: 70).

Si en 1964 identificar ciencia ficción y fantasía resultaba una provocación; en 1982, Ballard indica un marco que acota y corrige la explosión imaginativa en la creación artística $^{10}$. Es en este contexto, determinado por el equilibrio surrealista entre imaginación

\footnotetext{
${ }^{10}$ En una entrevista de 1986, abundaría en estos planteamientos: "I'd like to think that I work in the area of myths, dreams, legends, which seem to me to touch the real nature of our experience [...] Twenty, thirty, forty years ago, everybody had a clear idea that the world of reality was the external world around you: the world of work, and industry, commerce. The world of fantasy and imagination was the world inside our heads, our private dreams and hopes for the future. Now that's been reversed. Now the external landscapes of our world are almost entirely fictional, made up of advertising and publicity, an artificial man-made landscape. The only point of reality we each of us have is the point of reality inside our heads. Our own obsessions are all we have" (Ballard, 2014d: 227-228).
} 
y crítica, y por la concepción de una ciencia ficción del Inner Space, donde se sitúa la idea ballardiana de mito:

Me interesa ese conjunto de mitologías que no están vinculadas estrictamente con el pasado [...] Busco una mitología que comience ahora, en esta época, y que se lance hacia adelante. Y libros como Crash y La exhibición de atrocidades, además de las historias de Mitos del futuro próximo [...] son tentativas que procuran hacer frente a la corriente continua de eventos que rodea nuestra vida. Es una mitología que se apoya fundamentalmente en la ciencia y la tecnología, y también en el paisaje de las comunicaciones (Ballard, 2015: 106).

Pero esta mitología del futuro tiene un efecto bisagra: abre un tiempo nuevo mientras cancela el anterior: "Me interesan las mitologías que dibujan una línea terminal, en el fondo de la experiencia humana, las mitologías que representan los estados finales" (Ballard, 2015: 143).

Los mitos ballardianos parten de obsesiones una y otra vez revisadas, convertidas en metáforas abiertas, mitemas de una mitología interior que se proyectan sobre los paisajes surrealistas: "Presumably all obsessions are extreme metaphors waiting to be born. The whole private mythology, in which I believe totally, is a collaboration between one's conscious mind and those obsessions that, one by one, present themselves as stepping stones" (Ballard, 2014d: 182). De este modo, las playas desérticas, los hoteles abandonados, las piscinas vacías o las redes de autovías en torno a las grandes ciudades se convierten en espacios mentales abstractos en los que se despliegan las violentas psicomaquias ballardianas ${ }^{11}$. La combinación de estos mitemas conforma un nuevo mito del futuro: la posibilidad disolvente de la entropía como ocasión problemática de un nuevo comienzo. De este modo, reúnen los requisitos del mito, tal como expone José Manuel Losada:

Every myth is made up of a limited series of constitutive elements, also known as invariants or mythemes. These elements do not necessarily coincide with the plotline of the original myth, but rather give shape to its mythic essence. These unvarying elements have a mythic core, that is, they follow the logic of myth: they hold, in some way, a transcendent dimensión (Losada, 2015: 29).

\footnotetext{
${ }^{11}$ Entendemos estos motivos como mitemas en la obra de Ballard, y no tópicos, porque actúan como átomos míticos de naturaleza estructural o esquemática. Como observa Gilbert Durand, el mitema puede ser un tema, un motivo, un decorado mítico; y actuar de forma patente (repetición explícita de sus contenidos, situaciones, personajes y emblemas); o latente, por la repetición de su esquema intencional implícito (Durand, 2013: 344-345). Ambas formas se presentan sostenidamente en la obra de Ballard. Para su dimensión mítica, puede verse Brigg (1985: 87, 99, 110); y Gasiorek (2005: 8).
} 
Myths of the Near Future (1982) es una colección de diez relatos ${ }^{12}$, escritos entre 1976 y 1982. Myths... supuso para el autor el retorno a la ciencia ficción pura y una revisión de su narrativa de los años cincuenta (Ballard, 2014d: 139).

Durante ese periodo (1979-1982) publica también las novelas The Unlimited Dream Company (1979) y Hello America (1981). Se trata de una etapa de transición entre los experimentos de La exhibición de atrocidades (1970) y Crash (1973) y las novelas autobiográficas Empire of the Sun (1984) y The Kindness Of Women (1991).

El corpus formado por las novelas y el libro de relatos editados entre 1979 y 1982 evidencia un momento de cambio en los temas y las formas de la escritura ballardiana en el que es posible reconocer tanto la revisión de subgéneros del pasado, como el adelanto de algunos símbolos que determinarán su narrativa posterior.

En cuanto a las dos novelas, The Unlimited Dream Company es, tal vez, la más interesante. Por una parte, enlaza con las novelas autobiográficas posteriores, por cuanto supone una ficcionalización fantástico-delirante de aspectos de su vida, si bien en el entorno realista de Shepperton (Wilson, 2017: 100, 109). Los tres primeros capítulos presentan un personaje y unas situaciones típicamente ballardianos, hasta el punto de que podemos considerarlos como un desarrollo del relato "Notes toward a Mental Breakdown"13: un personaje obsesionado con hombres voladores; el mesianismo; impulsos homicidas contra su novia; el robo de un avión que termina accidentado... Sin embargo, a partir del accidente, la novela se convierte en un relato alucinatorio de tono mítico, en el que el protagonista cree ser un dios-pájaro con la misión de salvar a los habitantes del suburbio (Ballard, 2014b: 77-79). Se trata de un texto rebosante de erotismo degradado y místico a un tiempo, delirios violentos, alteraciones de la personalidad, canibalismo y dislocación del continuo espacio-tiempo (otra constante en Ballard desde "The Voices of Time"). La novela apela al surrealismo, desde el onirismo enmarcado en el contexto realista del accidente de avión, la influencia psicoanalítica, la concepción de un mundo determinado por las leyes del deseo, incluso la alusión a dos grandes mitos del surrealismo: Loplop, el dios-pajaro alter ego de Max Ernst, clara referencia del diospájaro en el que se convierte el protagonista; y quizá, más sutilmente, a los grandes transparentes de Breton ${ }^{14}$.

The Unlimited... es el texto ballardiano en el que mejor se lee la concurrencia de fantasía y ciencia ficción: una fantasía ilimitada, generadora de nuevos mitos, donde se funden Ícaro, Loplop y la redención mesiánica con una desbocada pulsión sexual y una

\footnotetext{
${ }^{12}$ Los títulos son los siguientes: "Myths of the Near Future", "Having a Wonderful Time", "A Host of Furious Fancies", "Zodiac 2000", "News from the Sun", "Theatre of War", "The Dead Time", "The Smile", "Motel Architecture", "The Intensive Care Unit".

${ }^{13}$ Ballard escribió "Notes toward..." en 1976, aunque no lo publicó hasta 1990 en War Fever. Dividido en dieciocho secciones, "Notes toward..." narra en el tono neutral de un informe clínico los hechos que llevan a la muerte de una mujer en un hotel de aeropuerto. El relato retoma situaciones, personajes y contextos propios de The Atrocity Exhibition. De hecho, el título aparece como tal en el capítulo V de The Atrocity... 14 "I guessed that I could use the population of Shepperton, not merely as part of my plan to escape from the town and deny finally that death from which I have already once escaped, but to make my challenge against the invisible forces who had given these powers to me" (Ballard, 2014b: 116).
} 
mirada satírica al extrarradio londinense; todo ello desplegado en marcos de sucesiva concreción: la relación problemática con la tecnología del personaje psicótico de los tres primeros capítulos; lo fantástico, que ocupa la mayor parte de la novela, como sueño del personaje agonizante o quizá ya muerto; y finalmente, la apelación al presente, el desocultamiento de la vida marcada por el consumismo y la alienación de los vecinos de Shepperton y su necesidad de ser redimidos. Los viejos mitos de Ballard — la imaginación pornográfica, la fantasía de volar y los accidentes de avión, los celos homicidas y el colapso mental — van abriendo paso a mitos nuevos: la ficcionalización de la propia vida, y la sátira social de un mundo aburrido y apático, ya vislumbrado en Vermilion Sands, si bien presentado en un contexto realista próximo.

Con frecuencia, en los libros de relatos de Ballard, el relato título asienta el concepto que rige el volumen. Se trata de textos seminales, con imágenes, modelos de personajes, símbolos, esquemas formales y tramas que permanecen en la escritura ballardiana. Por otra parte, son relatos de condensación, que reajustan y recrean, con variaciones, la preexistente y compleja mitología del autor ${ }^{15}$. Todo esto arroja una literatura obsesiva que perfila un universo cerrado, en el que lo nuevo siempre termina regresando a lo conocido; una literatura autorreferencial y laberíntica hecha de repeticiones y variantes.

En Myths of the Near Future reconocemos el universo simbólico madurado desde "The Terminal Beach" (1964) hasta Crash; desde el trauma de la guerra mundial hasta la psicopatología de los accidentes de tráfico, pasando por la obsesión por los medios de comunicación, el asesinato de Kennedy y la cultura pop americana. En este sentido, Ballard confesaría a Linda S. Kauffman: "In America, you see the future in its primitive, prehistoric state" (Kauffman, 1998: 166). Es decir, estos mitos de la cultura contemporánea americana se conforman como pasado del futuro, un tiempo que para el futuro próximo será su pasado fundacional. Este pasado es algo así como lo monstruoso que el mito, en sentido estricto, siempre ubica en un pretérito amargo, pero ya superado (Blumenberg, 2003: 24). El horror de la era atómica, la guerra mundial y los campos de exterminio; la locura de The Atrocity o el sadismo de Crash son ya el pasado intranquilizador, absoluto, del que arranca el nuevo tiempo mítico. Este tiempo es el futuro. Si el pasado se cerraba con la locura sexual y los accidentes de tráfico, el futuro se abre con el sueño escapista y redentor de volar: los hombres-pájaro y las avionetas siniestradas.

En esto surge la paradoja: por una parte, la imposibilidad de fijar unos mitos estables en un tiempo que está en continua transformación; por otra, la repulsión mutua entre progreso y mito: "Querer proyectar el mito en el esquema de progreso sería dar una actualidad superficial al mismo [...] El mundo va perdiendo monstruos. Se va convirtiendo en algo "más amable"” (Blumenberg, 2003: 127). Pero Ballard sortea estas

\footnotetext{
${ }^{15}$ Recordemos brevemente los títulos de los relatos que dan nombre a estos volúmenes y su importancia en la trayectoria narrativa del autor (excluimos los libros que son antologías de relatos ya publicados en libro anteriormente): "The Voices of Time", "The Terminal Beach", "The Day of Forever", "Low Flying Aircraft", "Myths of Near Future" y "War Fever".
} 
dificultades renunciando al futuro porque este es un presente indefinido; los monstruos o no se han ido o siempre regresan. Pero no debe olvidarse, por otra parte, que la ironía produce un efecto delimitador que inserta el mito en el esquema de un género distinto. En este sentido, Brigg observa: "Ballard's 'new myths' are characterized by this wedding of irony and myth and by their being set in the technological world" (Brigg, 1985: 89).

De los diez relatos, dos están escritos en tercera persona ${ }^{16}$; cinco en primera $^{17}$; y tres presentan formas mixtas: "Zodiac 2000" introduce una nota del autor y doce fragmentos con epígrafes; "Theatre of War" se compone de un prefacio del autor y dos partes divididas en transcripciones de programas de televisión, reportajes y entrevistas; "News from the Sun" presenta una narración en tercera persona que intercala pasajes del diario del protagonista.

En el relato "Myths of the Near Future" (Ballard, 2014a: 602-634), parece que el autor quisiera convertir en mito su propio universo literario. Al igual que sucede con "News from the sun" y "Memories of the Space Age", el relato trata del final de la era espacial. En este sentido, dice Peter Brigg: "The central concern is that man has either violated some essential structural aspect of nature by going into space or that it has marked some stage, not necessarily a positive one, in the process of evolution" (Brigg, 1985: 89).

Lo mismo encontramos en "News from the Sun", el fin de la carrera espacial y el ocaso de sus viejos mitos:

By leaving his planet and setting off into outer space man had committed an evolutionary crime, a breach of the rules governing his tenancy of the universe, and of the laws of time and space [...] Certainly the unhappy lives of the astronauts bore all the signs of a deepening sense of guilt. The relapse into alcoholism, silence and pseudo-mysticism, and the mental breakdowns, suggested profound anxietes about the moral and biological rightness of space exploration (Ballard, 2014a: 544-545).

Ballard recurre al mito de Prometeo para explicar el fin de la carrera espacial en el dramático final de "The Dead Astronaut", convirtiendo un Cabo Cañaveral abandonado en un espacio visionario, inspirado por Ernst y Delvaux, en el que se enfrenta el recurrente triángulo amoroso ballardiano, en el contexto de una rara enfermedad del espacio (Ballard, 2014a: 606-607).

El relato incorpora la intuición de la metáfora como instrumento mítico para cambiar la percepción del espacio y el tiempo. Se trata de una idea que ya aparecía en el lejano "The Voices of Time", pero que ahora se expresa más claramente: "What Elaine and the other victims were trying to do was to explore space, using their illness as an extreme metaphor with which to construct a space vehicle. The astronaut obsession was the key" (Ballard, 2014a: 608). Los mitos futuros se proyectan como metáforas, palabras eficaces que alteran la realidad a partir de estados depresivos, equivalentes a la entropía de los

\footnotetext{
16 "Myths of the Near Future" y "Motel Architecture".

17 "Having a Wonderful Time"; "A Host of Furious Fancies"; "The Dead Time"; "The Smile"; y "The Intensive Care Unit".
} 
cuerpos físicos (Ballard, 2014d: 206); que se articulan como ritos de repetición, en una prolongación del pensamiento mítico sobre los paisajes en ruinas del fin de la era espacial: "Perhaps, for the central nervous system, space was not a linear structure at all, but a model for an advancec condition of time, a metaphor for eternity which they were wrong to try to grasp" (Ballard, 2014a: 608-609). La metáfora posibilita una experiencia del espacio-tiempo en la que estos se reestablecerían para siempre (Ballard, 2014a: 621), simultaneando todos los yo temporales del protagonista, formando la ilusión de una figura alada por el solapamiento de todos los cuerpos con sus piernas y brazos como un inmenso plumaje eléctrico (recuérdese la simbología escapista del hombre-pájaro desde The Unlimited...), pero que, ahora, se presenta acompañada de una coartada científico-lógica, lo que desplazaría la imagen de la alucinación fantástica a la ciencia ficción: "An illusion, of course, a blur of multiple selves that simmered from his arms and shoulders, like an inmense electric plumaje. But perhaps at some time in the future he became a winged man, a glass bird" (Ballard, 2014a: 621-622), como sucede en las fotografías surrealistas de Étienne J. Marey, aludidas en el relato.

Escrito un año antes, "News from the Sun" es prácticamente un borrador de "Myths...". También se desarrolla en un Cabo Cañaveral en el fin de la carrera espacial, considerada como una hybris que debe ser purgada con una enfermedad que afecta a la percepción del tiempo y el espacio. En ambos, la decadencia de un paisaje cargado de simbolismo activa mutaciones imprevisibles, en forma de regresión hacia etapas premíticas: "In Ballard's fiction, dramatic changes in the natural environment reawaken the most primal, pre-social, even pre-human memories in living humans, resituating them in a time before myth" (Parkinson, 2015: 187).

En ambos relatos aparecen otros mitemas ballardianos: el triángulo formado por el protagonista, su mujer y un médico, la aviación, la pornografía, la pintura surrealista, la promesa del paraíso terrenal y la fantasía de los hombres-pájaros. Pero las estrategias retóricas son diferentes. En "News..." se vacila en el modo narrativo entre la tercera persona de un narrador externo perturbado y la primera del diario del protagonista. Si en "Myths..." el acceso al nuevo mito es metafórico; en "News..." es ritual a través de la construcción de pequeños santuarios que, a modo de catálogos, actúan como "relics of a yet to be experienced future" (Ballard, 2014a: 539). La resolución es distinta: en "News" el protagonista queda a la espera de las noticias del sol; en "Myths..." se produce (en la mente dañada del personaje) la redención mesiánica de la humanidad, en un sentido cercano a The Unlimited...

Este es, a nuestro juicio, el mito fundacional del futuro ballardiano: el miedo a la redención en el espacio entrópico generado por la nueva ciencia ficción. Si los mitos de Prometeo, Ícaro y Proteo están presentes en esta etapa de transición, Myths... anuncia un ciclo narrativo caracterizado por la disección de la sociedad postmoderna. Se trata de una proyección hacia el futuro inmediato de la atención del autor que, quizá descargando la experiencia traumática del pasado en sus novelas autobiográficas, deja para la ficción no directamente autorreferencial el examen de la realidad inminente a partir de categorías 
nuevas. Esta encrucijada mítica, todavía con muchos rasgos de ciencia ficción, dará paso a novelas aparentemente realistas, desarrolladas en mundos urbanos cerrados (Running Wild, Millenium People, Kingdom Come, Cocaine Nights y Super-Cannes).

La aspiración surrealista de rehacer el mundo, fundiendo lo sagrado mítico con lo profano cotidiano (Gasiorek, 2005: 10-11), a través de la experiencia transformadora y alucinatoria de un héroe imbuido en una psicosis mesiánica da lugar a un nuevo $\log _{0}{ }^{18}$ que articula su última narrativa en la que la redención adopta su aspecto más perturbador, anunciando la presencia de nuevos monstruos sociales que la imaginación mítica debe conjurar: el consumismo, el aburrimiento y la violencia colectiva de las últimas novelas de Ballard ofrecen una prospectiva siniestra y desoladora de los espacios suburbanos, los centros comerciales y las urbanizaciones residenciales. Estas patologías sociales se intuían como síntomas de la enfermedad del espacio en "Myths..." y "News...", incluso en la beach fatigue que aquejaba a los habitantes de Vermilion Sands.

En tales escenarios, la redención ya no es posible o adopta formas abyectas, soluciones descabelladas en las que el gurú sádico que había encarnado Vaughan en Crash se presenta con un nuevo discurso mesiánico y fascista, que es, a un tiempo, regresión y profecía. Así lo expresa el personaje de Wilder Penrose en Super-Cannes:

Homo sapiens is a reformed hunter-killer of depraved appetites, which once helped him to survive. He was partly rehabilitated in an open prison called the first agricultural societies, and now finds himself on parole in the polite suburbs of the city state. The deviant impulses coded into his central nervous system have been switched off. He can no longer harm himself or anyone else. But nature sensibly endowed him with a taste for cruelty and an intense curiosity about pain and death. Without them, he's trapped in the afternoon shopping malls of a limitless mediocrity. We need to revive him, give him back the killing eye and the dream of death (Ballard, 2017: 289-290).

La regresión mítica liberadora de The Unlimited... y "Myths..." se convierte en el logos pregnante del nuevo milenio en el que el mesías ya no es el hombre-pájaro de las alucinaciones de un héroe fallido sino el führer de las fantasías totalitarias de un psiquiatra de suburbio o de polígono tecnológico (Ballard, 2014c: 106).

La dimensión prospectiva, la exploración del Inner Space y la relación problemática con la tecnología (entendida ahora como ingeniería social) incorporan modos expresivos alejados de la ciencia ficción clásica y de los delirios surrealistas del periodo de transición. En Myths... encontramos un relato que, de forma embrionaria, adelanta este nuevo espacio de la ciudad vacacional y el fracaso de su redentor: "Having a Wonderful Time", en el que un resort de lujo se convierte en un campo de vacaciones para retener secretamente a los parados, nueva variante de la beach fatigue.

La narración en primera persona de "A Host of Furious Fancies" pone en marcha un juego irónico en el que se desmonta el artificio retórico del punto de vista único, mediante

\footnotetext{
${ }^{18}$ Anotemos con Blumenberg que el mito es ya un trabajo del logos (Blumenberg, 2003: 20). De este modo, el universo de las imágenes simbólicas ballardianas se mueve en una espiral determinada por la tensión fundante entre mito y logos.
} 
sendos finales en los que se evidencian las fisuras epistemológicas de esta forma narrativa. La lectura avanza una sospecha creciente hacia el narrador o bien es sorprendida al final con una revelación que destruye lo contado/leído. "A Host..." es un relato fantástico incorporado a una narración realista en la que los límites de ambos se difuminan: el cuento infantil de la Cenicienta que obsesiona a la protagonista y la interpretación psicoanalítica del psiquiatra que dice haberla curado y que trata de imponer un punto de vista que se deshará en el contrapunto final, dejando al lector sumido en la perplejidad.

"Motel Architecture" es un relato de crímenes a partir de la obsesión con la escena de la ducha de Psicosis (Alfred Hitchcock, 1960). Comparte con "A Host..." su carácter hipertextual: ambos reescriben total o parcialmente las obras a las que aluden: $L a$ Cenicienta y Psicosis. "Motel Architecture" enlaza no solo con el film de Hitchcock, sino también con el fragmentarismo mítico de The Atrocity... Como en esta, encontramos el despliegue de un pensamiento metafórico surrealista que relaciona lo orgánico y lo inorgánico, la geometría del espacio con el cadáver del film, en la intuición del secreto (constante en la obra de Ballard) del tiempo y el espacio, aunando el modo de conocimiento mítico con el pensamiento científico:

The extraordinary relationship between the geometry of the shower stall and the anatomy of the murdered woman's body seemed to hold the clue to the real meaning og everything in Pangborn's world, to the unstated connections between his own musculature and the inmaculate glass and chromium universe of the solarium. In his headier moments Pagborn was convinced that the secret formulae of his tenancy of time and space were contained somewhere within this endlessly repeated clip of film (Ballard, 2014a: 503).

La estructura narrativa del relato descansa, como en tantas ocasiones, en la tensión producida por la coexistencia de un narrador en tercera persona y un focalizador personaje con la percepción y el razonamiento alterados.

"The Smile" y "The intensive care unit" son dos relatos ejemplares que despliegan narrativamente la hipótesis de la soledad y el miedo al otro en la sociedad contemporánea: el primero en un contexto estilizadamente contemporáneo; el segundo en un futuro distópico en el que la tecnología sustituye o media en las relaciones humanas. El relato se equilibra entre la sátira de las relaciones mediadas por la tecnología, con sus equívocos y frustraciones, y la regresión violenta y psicopática, no exenta de contradicción, desatada por el contacto físico entre los personajes.

"Theatre of War" parodia el lenguaje de los informativos de televisión, transcribiendo noticiarios reales de la guerra de Vietnam (Ballard, 2014d: 194). Se trata de una variante de "The Killing Ground" (1969). El prefacio del autor plantea una narración nuevamente ejemplar, respuesta a la hipótesis de una guerra civil en el Reino Unido.

Sin embargo, el relato más decisivo en la creación de estos mitos del futuro próximo es "Zodiac 2000". Se trata de una nueva muestra del talento de Ballard para la psicogeografía: al espacio realista de la clínica, las autovías, los clubes de sexo, el hotel, etc. 
se superpone el espacio mental de las casas zodiacales, de tal modo que encontramos, de nuevo, la intersección de lo fantástico con lo real, pero ahora al servicio de una alegoría perturbadora en la que concurren ciencia ficción (universos paralelos, mundos alternativos) y sátira social (consumismo, obsesión tecnológica) en un relato entrevisto por un personaje paranoico. Como sucedía en The Atrocity..., es el mundo de los objetos y de los acontecimientos, más que los personajes, lo que articula la estructura narrativa del relato (Ballard, 2014d: 77). Precedido de una nota del autor, el relato está dividido en doce fragmentos con epígrafes que corresponden a los nuevos signos del zodiaco. La fórmula remite a las novelas condensadas ${ }^{19}$ de The Atrocity... si bien en este caso Ballard ha arriesgado menos tanto en el aspecto formal como en la comprensibilidad del texto. "Zodiac 2000" es un relato difícil y con numerosos aspectos desconcertantes, pero conserva el principio de no contradicción, cosa que deliberadamente se fractura en The Atrocity... La breve nota del autor informa de la necesidad de actualizar el zodiaco de acuerdo con el nuevo horizonte tecnológico: "The houses of our phychological sky are no longer tenanted by rams, goats and crabs but by helicopters, cruise missiles and intrauterine coils, and by all the spectres of the psychiatric Ward [...] Here, anyway, is an s-f zodiac, which I asume the next real one will be" (Ballard, 2014a: 493). Las máquinas se convierten en las contrapartidas de los viejos signos zoomórficos del zodiaco.

Pero antes de examinar el proceso de construcción de estos nuevos signos, debemos detenernos en la estructura del relato y en el modo en que se relacionan los elementos de ciencia ficción con los fantástico-míticos. A pesar de su oscuridad, podemos distinguir tres tramas narrativas concéntricas. La primera, de carácter realista, puede resumirse del siguiente modo: un enfermo mental, que cree provenir de un universo alternativo más vigoroso, se escapa del sanatorio mental en el que está recluido desde hace años; asesina a una mujer y es devuelto al centro y sometido a aislamiento, quedando en un estado cercano a la catatonia. La segunda es un relato de ciencia ficción que corresponde al delirio alucinatorio del enfermo. Este cree que ha sido secuestrado por una red terrorista con el objeto de provocar la tercera guerra mundial (otro de los mitemas de Ballard). En el desarrollo de esta conspiración, el protagonista se ve envuelto en una serie de actividades violentas y sexuales con dos personajes: Heller, que responde al tipo de sádico ballardiano en la línea de Vaughan (Crash) con el que comparte aspectos como las cicatrices, el sexo con prostitutas en vehículos y el crimen psicótico; y Renata, a la que estrangulará; que comparte con la Renata de Crash no solo el nombre sino también la hipersexualidad, y la percepción del cuerpo como un collage erótico. En esta línea argumental encontramos una mezcla de ciencia ficción (universos paralelos, tecnología problemática), thriller terrorista (secuestros, asesinatos, amenazas nucleares) y pornografía sádica. Finalmente, la tercera trama es fantástico-mítica. El enfermo que cree vivir esta trama de ciencia ficción, la experimenta en su Inner Space como un viaje de perfeccionamiento espiritual y liberación a través de las doce casas zodiacales, que

${ }^{19}$ Para el concepto de novela condensada, véase Ballard (2014d: 52). 
concluye con el vuelo transformador tan frecuente en el Ballard posterior a The Unlimited...: "Now he would leave them, and take the left-handed staircase to the roof above his mind, and fly away across the free skies of his inner space" (Ballard, 2014a: 501). Ballard narra la historia que cree vivir el enfermo y también cómo la vive, es decir, su experiencia, objetivada a través de las casas zodiacales, cumpliendo la finalidad expuesta en su nota: crear un zodiaco de ciencia ficción para el presente, es decir, para el futuro.

Con estas premisas, los doce textos que componen "Zodiac 2000" desarrollan paralelamente las tres líneas argumentales, en un relato en tercera persona, pero, como en ocasiones anteriores, recurriendo al protagonista como focalizador. El orden de los epígrafes corresponde al de las casas del zodiaco, por lo que es sencillo determinar a qué signo convencional corresponde cada uno de los nuevos, si bien a veces resulta difícil determinar la conexión entre ambos.

El primero, "The Sign of the Polaroid", asocia esta cámara fotográfica con el signo de Aries. La explicación está adelantada por el autor: "As for the ram, that tireless guardian of the domestic flock, his counterpart in our own homes seems to be the Polaroid camera, sheperding our smallest memories and emotions, our most tender sexual acts" (Ballard, 2014a: 493). Se trata por tanto de una metáfora analógica entre cuatro términos: Aries es al rebaño, lo que la polaroid a la vida íntima. Hay en esta analogía un elemento irónico: Aries guarda y protege; la cámara conserva y revela. El enfermo, que ve que lo fotografían y observan, no se siente protegido, sino perturbado y amenazado, activando así su deseo de huir.

"The Sign of the Computer" corresponde al signo de Tauro. De nuevo la dificultad de la asociación obliga a Ballard a intervenir en la nota del autor: "Perhaps our true counterparts of these workaday creatures are the machines which guard and shape our lives in so many ways -above all the taurean computer, seeding its limitless possibilities" (Ballard, 2014a: 493). Se trata del ordenador MIT'S ULTRAC 666, presentado como la computadora más poderosa del mundo. En el fragmento, el doctor Rotblat revela a la prensa que el protagonista parece haber nacido en un universo alternativo simétrico. Rotblat asume una función similar a la del Dr. Nathan en The Atrociy..., con las diferencias que se expondrán más abajo.

"The Sign of the Clones" alude al signo de Géminis. El fragmento reúne algunos de los temas y recursos expresivos más conocidos de Ballard: los universos alternativos, la música temporal de los quásares, el perspectivismo, las preguntas que el narrador hace prestando su voz al personaje, y el catálogo.

“The Sign of the IUD” [DIU] corresponde al signo de Cáncer. La asociación metafórica se basa en la semejanza. La acción sexual entre el protagonista y la mujer que lo ha ayudado a escapar da lugar a imágenes que remiten, de nuevo, a The Atrocity...: "Through the glass door he could see her blurred thighs and breast, as if distorted by some computer permutating all the possibilities of an alternative anatomy" (Ballard, 2014a: 495). En este pasaje se pone en marcha la trama de thriller tecnológico de ciencia ficción. 
Paralelamente, Ballard introduce la vivencia alucinatoria de lo que, como se ha explicado, es ya de por sí una alucinación. Así la descripción del DIU como una doble esvástica, da lugar a una de las preguntas focalizantes habituales en el autor: "Was it a clue left for him, a modulus to be multiplied by everything in this right-handed world - the contours of this Young woman's breast, the laws of chemical kinetics, the migration song of swallows?" (2014a: 496), lo que da paso a la fantasía zodiacal como experiencia del Inner Space: "After the camera, the computer and the clones, the coil was the fouthr house of that zodiac he had already entered, the twelve-chambered mansión through which he must move with the guile of a master-burglar" (2014a: 496).

"The Sign of the Radar Bowl" sustituye al signo de Leo. La operación de sustitución reviste un particular interés. Sobre la sobrecapacidad auditiva del personaje, dice lo siguiente: "he could [...] hear the answering over-the-horizon probes of the Russian sites near Murmanks, distant lions roaring their fear at each other, marking their claims to imposible territories". La sustitución obedece a una doble metáfora y una sinécdoque: el signo del radar desplaza al signo de Leo, porque el radar es como el oído del personaje que oye las sondas rusas que son como los rugidos de los leones: en virtud de la metonimia el productor (Leo) sustituye a la cosa producida (el rugido).

"The Sign of the Striper" es el nuevo signo de Virgo. En principio puede entenderse que la sustitución se funda en la ironía. Más aún si se considera que el episodio tiene por común denominador el sexo: el espectáculo pornográfico de Renata en un club y el sexo de Heller con prostitutas en el asiento trasero de su coche. El propio personaje se pregunta a través del narrador, en uno de esos mecanismos de interrogación frecuentes en Ballard: "But were they unconsciously giving him the keys to the sixth house?"; y la respuesta da la clave de este signo zodiacal: "In the codes of Renata's body, in the junction of nipple and finger, in the sulcus of her buttocks, waited the possibilities of a benevolent psychopatology" (2014a: 497-498). Por esto la striper es la nueva virgo, porque aún las posibilidades de una psicopatología benevolente están sin explorar. Es decir, la aparente ironía se deshace en una nueva analogía de cuatro términos: el signo de Virgo es a la virginidad lo que el signo de la striper es a las inexploradas posibilidades psicopatológicas del cuerpo de Renata. La posibilidad de una psicopatología benevolente, nuevo mito para un futuro próximo, es uno de los temas investigados por los personajes de Ballard en sus últimas novelas.

"The Sign of the Psychiatrist" desplaza al de Libra, en virtud de su sencilla analogía: ambos buscan el equilibrio. El Profesor Rotblat, encarnación del Dr. Nathan de The Atrocity... y adelanto del Dr. Penrose (Super-Cannes) afirma que el actual papel del psiquiatra no es curar al paciente, sino reconciliarlo con sus fortalezas y debilidades. La diferencia con Penrose y otros personajes similares de la última etapa de Ballard estriba en que su voz queda silenciada por las alucinaciones del protagonista.

"The Sign of the Psycopath" corresponde al signo de Escorpio. El centro de este fragmento es Heller, psicópata torturador y asesino, que planea desencadenar la tercera guerra mundial. La analogía es obvia: las naturalezas dañinas del escorpión y Heller. 
"The Sign of hypodermic" sustituye a Sagitario. El pasaje enlaza con el tratamiento de choque recibido en la clínica por el protagonista, mientras que este compone el relato paranoico de la conspiración, y cree ser objeto de un experimento científico. Es importante señalar la función de la televisión en este fragmento, acogiendo la influencia de Macluhan y adelantándose en su planteamiento a la película Videodrome de David Cronenberg (1983): "Multiplied by the ULTRAC computer, the wave-functions of his hallucinating brain would be transmitted on the nationwide channels and provide a new set of operating formulae for their passage through consciousness" (2014a: 499).

"The Sign of the Vibrator" desplaza al signo de Capricornio. De nuevo la relación entre ambos signos parece oscura. El protagonista observa cómo Renata se masturba con el vibrador, y piensa que cuando las televisiones retransmitan este acto los curiosos asumirían el trampantojo de que ella estaba dando a luz a la máquina, como una hija de su matrimonio con las impresiones del MIT'S ULTRAC 666, el ordenador que representaba al signo de Tauro. Se trata a nuestro juicio de la imagen más poderosa del relato: la retransmisión del parto de la mujer que da a luz al artilugio erótico hijo de su relación con el ordenador.

"The Sign of the Cruise Missile" corresponde al signo de Acuario. Mientras espera el lanzamiento de misiles que dará comienzo a la guerra mundial, tras estrangular a Renata, el protagonista mira la televisión que hace las veces de acuario. De nuevo nos encontramos ante una metáfora compleja formada por una asociación casi arbitraria: el misil sustituye a Acuario porque es en lo que piensa el personaje.

Finalmente "The Sign of the Astronaut" desplaza al signo de Piscis. A través del cristal de la sala de aislamiento (como un pez en la pecera), el protagonista mira a los doctores y se da cuenta del engaño. La historia de ciencia ficción se ha disipado en la realidad de la reclusión en la clínica mental. Sin embargo, la historia fantástica del viaje a través de las casas del zodiaco se confirma: escapa a través de la muerte de Renata: "Now he would leave them, and take the left-handed staircase to the roof above his mind, and fly away across the free skies of his inner space" (2014a: 501).

\section{CONCLUSIONES}

La obra de Ballard obliga a modificar los parámetros genéricos de la ciencia ficción clásica, especialmente el novum de carácter científico, la dimensión prospectiva y su carácter realista, en beneficio de un género más atento a la experiencia humana del progreso científico-tecnológico, a la prospectiva del presente y a una retórica expresiva que responda a la nueva verosimilitud que este exige.

En este sentido, la frontera entre ciencia ficción y género fantástico se hace habitable, permitiendo la relación, aunque no la identificación, entre la primera y la segunda. En tal contexto es posible hablar de relación entre mito y ciencia ficción.

El mito del futuro ballardiano debe entenderse de tres modos: como la configuración imaginaria de las obsesiones patológicas de sus personajes, con una proyección externa 
capaz de dibujar paisajes de alta carga simbólica; como recurso retórico que dispone espacios fantásticos dentro de los esquemas peculiares de su comprensión de la ciencia ficción como prospectiva del presente; y como tiempo original, del origen, en el que puedan constituirse estos mitos futuros y asegurarse su perdurabilidad.

Myths of the Near Future representa un producto acabado y sobresaliente de las posibilidades teóricas anteriormente señaladas. A lo largo de los relatos que lo componen, y en consonancia con las obras escritas en esta etapa, Ballard fortalece los nuevos mitemas de su escritura, al tiempo que revisa los anteriores, creando nuevos modelos combinatorios entre ellos y perfilando nuevos mitos personales en forma de metáforas, símbolos, objetos y espacios que desplegarán sus posibilidades en su trayectoria literaria ulterior.

\section{REFERENCIAS BIBLIOGRÁFICAS}

AlAZRAKI, J. (2001). “¿Qué es lo neofantástico?”. En Teorías de lo fantástico, D. Roas (ed.), 265-282. Madrid: Arco / Libros.

Angenot, M. (1979). "The Absent Paradigm: An Introduction to the Semiotics of Science Fiction". Science Fiction Studies 17, vol. 6, Part. 1. https://www.depauw.edu/sfs/backissues/17/angenot17.htm [13/09/2020].

BAllard, J. G. (1968). "Interview with J. G. Ballard. Munich Round Up", 100, 104-6. http://www.ballardian.com/munich-round-up-interview-with-jg-ballard [13/09/2020].

(1997). A User's Guide to the Millennium. Essays and Reviews. Londres: Harper Collins.

(2014a). The Complete Short Stories, Volume Two. London: Fourth State. (2014b). The Unlimited Dream Company. London: Fourth State. (2014c). Kingdom Come. London: Fourth State. (2014d). Extreme Metaphors. Collected Interviews. London: Fourth State. (2015). Para una autopsia de la vida cotidiana. Conversaciones. Buenos Aires: Caja Negra. (2017). Super-Cannes. New York: Picador.

BARRENECHEA, A. (1972). "Ensayo de una tipología de la literatura fantástica". Revista Iberoamericana $80,391-403$.

BAXTER, J. (2008). J. G. Ballard's Surrealist Imagination. Burlington, Ashgate: Spectacular Authorship.

BERARDi, F. (2014). Después del futuro. Desde el futurismo al ciberpunk. El agotamiento de la modernidad. Madrid: Enclave.

Blumenberg, H. (2003). Trabajo sobre el mito. Barcelona: Paidós. (2008). La legitimación de la Edad Moderna. Valencia: Pre-textos.

BRIGG, P. (1985). J. G. Ballard. West Linn, OR / Mercer Island, WA: Starmont House. 
Broderick, D. (1995). Reading by Starlight. Postmodern Science Fiction. London \& New York: Routledge.

CAMPRA, R. (2008). Territorios de la ficción. Lo fantástico. Sevilla: Renacimiento.

CAPAnNA, P. (2006). Idios Kosmos. Claves para una biografía de Philip K. Dick. Buenos Aires: Cántaro.

DurAnd, G. (2013). De la mitocrítica al mitoanálisis. México: Anthropos.

GASIOREK, A. (2005). J. G. Ballard. Manchester: Manchester University Press.

GreENLAND, C. (2012). The Entropy Exhibition. New York: Routledge.

Heinlein, R. (1959). "Science Fiction: Its Nature, Faults and Virtues". En The Science Ficition Novel. Imagination and Social Criticism, B. Davenportl (ed.), 14-48. Chicago: Adevent.

JAMESOn, F. (2005). Arqueologías del futuro. El deseo llamado utopía y otras aproximaciones de ciencia ficción. Madrid: Akal.

Kauffman, L. S. (1998). Bad Girls and Sick Boys. Fantasies in contemporary art and culture. Berkeley: University of California Press.

LEM, S. (1974). "Todorov's Fantastic Theory of Literature”. Science Fiction Studies 1, Vol. 1, Part. 1. https://www.depauw.edu/sfs/backissues/4/lem4art.htm [13/09/2020].

LosADA, J. M. (2015). “The Structure of Myth and Tipology of its Crisis". En Myths in Crisis: The Crisis of Myth, J. M. Losada \& A. Lipscomb (eds.), 3-32. Newcastle upon Tyne: Cambridge Scholars Publishing.

MeletinsKi, E. M., (2001). El mito. Madrid: Akal.

Moreno, F. A. (2009). "La ficción prospectiva: propuesta para una delimitación del género de la ciencia ficción". En Ensayos sobre ciencia ficción y literatura fantástica, T. López Pellisa y F. A. Moreno (eds.), 65-93. Madrid: Asociación Cultural Xatafi / Universidad Carlos III. (2010). Teoría de la Ciencia Ficción. Vitoria: Portal Editions.

Novell Monroy, N. (2008). Literatura y cine de ciencia ficción: perspectivas teóricas. Tesis Doctoral, Universitat Autònoma de Barcelona https://bibliocecifi.files.wordpress.com/2017/05/literatura-y-cine-en-cienciaficcic3b3n-perspectivas-tec3b3ricas-tesis-doctoral-noemc3ad-novellemonroy.pdf [13/09/2020].

PARKInson, G. (2015). "Surrealist Painting as Science Fiction: Considering J. G. Ballard's 'Innate Releasing Mechanism"'. En Surrealism, Science Fiction and Comics, G. Parkinson (ed.), 174-193. Liverpool: Liverpool University Press.

RoAs, D. (2011). Tras los límites de lo real. Una definición de lo fantástico. Madrid: Páginas de Espuma.

SÁNCHEZ FERNÁNDEZ, C. (2018). "Nihilism, self-transcendence and abjection in J.G. Ballard's Crash". Odisea 19, 89-107. http://ojs.ual.es/ojs/index.php/ODISEA/article/view/2261/3085 [13/09/2020]. 
SELLAR, S. (2007). "Future Fascination: Ballard in SFX", SFX Magazine, October $30^{\text {th }}$, http://www.ballardian.com/future-fascination-ballard-in-sfx [13/09/2020].

Suvin, D. (2016). Metamorphoses of Science Ficton. Bern: Peter Lang.

Todorov, T. (2001a). "Definición de lo fantástico". En Teorías de lo fantástico, D. Roas (ed..), 47-64. Madrid: Arco / Libros.

(2001b). "Lo extraño y lo maravilloso". En Teorías de lo fantástico, D. Roas (ed..), 65-81. Madrid: Arco / Libros.

WiLson, D. H. (2017). J. G. Ballard. Chicago / Springfield: University of Illinois Press.

Fecha de recepción: 15/01/2021

Fecha de aceptación: 27/04/2021 